\title{
Molecules in nearby and primordial supernovae
}

\author{
Isabelle Cherchneff and Simon Lilly \\ Institut für Astronomie, ETH Hönggerberg \\ Wolfgang-Pauli-Strasse, 16, 8093, Zürich, Switzerland \\ email: isabelle.cherchneff@phys.ethz.ch
}

\begin{abstract}
We present new chemical models of supernova (SN) ejecta based on a chemical kinetic approach. We focus on the formation of inorganic and organic molecules including gas phase dust precursors, and consider zero-metallicity progenitor, massive supernovae and nearby core-collapse supernovae such as SN1987A. We find that both types are forming large amounts of molecules in their ejecta at times as early as 200 days after explosion. Upper limits on the dust formation budget are derived. Our results on dust precursors do not agree with existing studies on dust condensation in SN ejecta. We conclude that PMSNe could be the first nonprimodial molecule providers in the early universe, ejecting up to $34 \%$ of their progenitor mass under molecular form to the pristine, local gas.
\end{abstract}

Keywords. Astrochemistry, molecular processes, supernova: general, individual (SN1987A), early universe

\section{Introduction}

Wether supernovae are major dust makers in the universe has been a long-standing debate. It was triggered by the explosion of SN1987A in the LMC two decades ago and the subsequent observation of dust and molecules like $\mathrm{CO}$ and $\mathrm{SiO}$ forming as early as 200 days after explosion. At high redshifts $(z \geqslant 6)$, dust has been proposed to explain the reddening of background quasars and Lyman $\alpha$ systems. At these early times, only very massive, evolved stars can be dust makers, due to stellar evolution time constraints. Some theoretical studies have tackled the problem of molecule formation in nearby core-collapse SNe (hereafter CCSNe, see Lepp et al. 1990, Liu \& Dalgarno 1994) or the condensation of dust in either CCSNe or primordial massive supernovae (PMSNe, see Kozasa et al. 1989, Clayton et al. 1999, Todini \& Ferrara 2001, Nozawa et al. 2003, Schneider et al. 2004). These studies assume steady-state from time scale analysis and are often based on an incomplete treatment of the chemical processes. As for dust formation, the formalism used is that developed for the homogeneous nucleation of water droplets in the Earth's atmosphere. This approach has been seriously questioned by Donn \& Nuth (1985) when applied to the formation of solids in circumstellar environments where equilibrium conditions do not apply and the condensation nuclei are often on molecular scale. We report in this proceeding preliminary results of a novel study on molecule and dust formation in supernova ejecta based on a chemical kinetic approach. We describe dust nucleation from the gas phase and the global ejecta chemistry using all chemical processes relevant to SNe ejecta. We then apply this chemical network to nearby CCSNe and PMSNe of zero-metallicity progenitor characteristic of Population III stars. Molecular contents and upper limits for dust yields are derived for these environments. 
Table 1. Ejecta parameters versus time after explosion for both CCSN and PMSN models.

\begin{tabular}{lcc}
\hline & CCSN & PMSN \\
\hline Progenitor mass $\left(\mathrm{M}_{\odot}\right)$ & 20 & 170 \\
Helium core mass $\left(\mathrm{M}_{\odot}\right)$ & 6 & 85 \\
$M\left({ }^{56} \mathrm{Co}\right)\left(\mathrm{M}_{\odot}\right)$ & 0.074 & 3.52 \\
\hline$T_{0}(100$ days $)(\mathrm{K})$ & 5000 & 21000 \\
$T(500$ days $)$ & 1490 & 1480 \\
$T(1000$ days $)$ & 889 & 470 \\
\hline$n_{0}(100$ days $)\left(\mathrm{cm}^{-3}\right)$ & $8.5 \times 10^{10}$ & $6.5 \times 10^{11}$ \\
$n(500$ days $)$ & $5.6 \times 10^{8}$ & $5.2 \times 10^{9}$ \\
$n(1000$ days $)$ & $7.0 \times 10^{7}$ & $6.5 \times 10^{8}$ \\
\hline
\end{tabular}

\section{Physical model of PMSN and CCSN ejecta}

We consider two surrogates of SN explosions: a $20 \mathrm{M}_{\odot}$ CCSN representative of SN1987A with metallicity typical of the LMC, and a $170 \mathrm{M}_{\odot}$ PMSN of zero-metallicity progenitor. The gas temperature $T$ is determined mainly by the explosion energy $\left(1 \times 10^{51}\right.$ ergs and $2 \times 10^{52}$ ergs for a typical CCSN and PMSN, respectively) and the CCSN surrogate $T$ profile is that of Kozasa et al. (1991). For the PMSN, we use the $T$ variation of Nozawa et al. (2003) for their $170 \mathrm{M}_{\odot}$ pair-instability SN. The ejecta expansion for both SN surrogates is homologous and the gas density varies with $t$ according to $n\left(M_{r}, t\right)=n_{0}\left(M_{r}, t_{0}\right) \times\left(t / t_{0}\right)^{-3}$, where $M_{r}$ is the mass coordinate and $n_{0}$ is the gas number density at $t_{0}$. Values for the various gas parameters are summarized in Table 1. The ejecta velocity for both surrogates is kept constant at $2000 \mathrm{~km} \mathrm{~s}^{-1}$ in good agreement with values derived from atomic emission lines in SN1987A and models from Schneider et al. (2004).

\section{The ejecta chemistry}

We are interested in studying the chemistry from time $t_{0}=100$ days to $t=1,000$ days, as molecules like $\mathrm{CO}$ and $\mathrm{SiO}$ and dust were observed over this time range in SN1987A (Spyromilio et al. 1988, Roche et al. 1991, Wooden et al. 1993). The light curve of SN1987A between 100 and 1000 days after explosion is dominated by the radioactive decay of ${ }^{56}$ Co. This decay produces $\gamma$-rays that are Compton-scattered and creates a fast, energetic electron population in the gas. Collisions with such electrons are among the main destruction processes to molecules. We assume that similar radioactivity-induced processes take place in the ejecta of PMSNe. Compton electron collisions proceed with rates given by Liu \& Dalgarno (1995) in the CCSN surrogate, whereas the rates are rescaled according to the ${ }^{56}$ Co mass produced over the mass cut for the PMSN case (Umeda \& Nomoto (2002). Evidence for strong mixing in SN ejecta is given by the early emergence of $\gamma$-rays from cobalt decay in SN1987A spectra (Pinto \& Woosley 1988). Therefore, we consider two extreme cases for both SN surrogates: a microscopically fully-mixed ejecta, where we allow for hydrogen penetration from the progenitor envelope and consequent mixing, and a stratified ejecta that retains the onion-like structure resulting from the progenitor nucleosynthesis. Mixing in SN ejecta is expected to be macroscopic rather than microscopic, with the existence of homogeneous clumps and knots resulting from Rayleigh-Taylor instabilities after explosion and observed in SN remnants such as Cas A (Douvion et al. 2001). For the fully-mixed SN1987A surrogate, elemental abundances are those of Kozasa et al. (1989) whereas the elemental compositions of Nosawa et al. (2003) are used for both the PMSN fully-mixed and unmixed cases. 


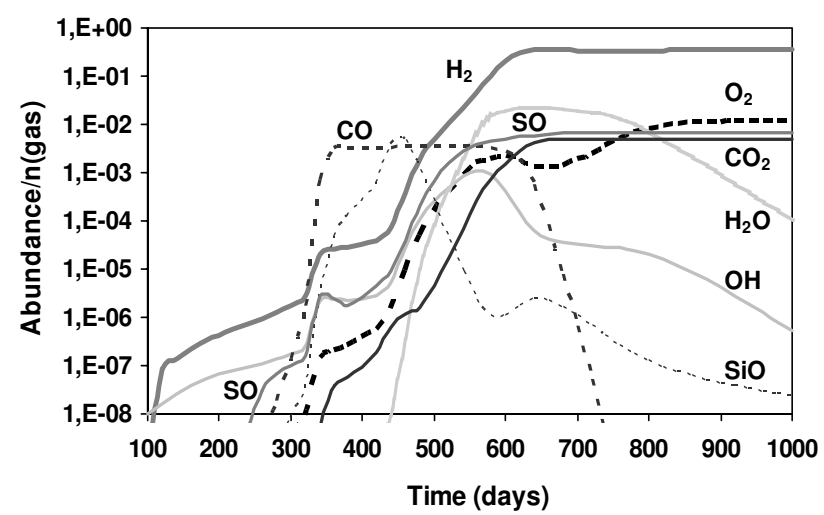

Figure 1. Molecular abundances versus time for the $170 \mathrm{M}_{\odot}$ PMSN fully-mixed case.

The chemical network includes tri-molecular reactions efficient in high density media, bi-molecular processes (neutral-neutral reactions with or without activation energy barriers, ion-molecule reactions) and radiative association reactions. In total, the system comprises 90 species and between 400 to 500 reactions, depending on the ejecta region under study. Among the chemical species, we consider astro-physically relevant molecules including $\mathrm{CO}, \mathrm{CO}_{2}, \mathrm{O}_{2}$, SO, $\mathrm{NO}, \mathrm{C}_{2} \mathrm{H}_{2}, \mathrm{H}_{2} \mathrm{O}$ and $\mathrm{H}_{2}$ and dust precursors (chains, rings and clusters) such as $(\mathrm{MgO})_{n},\left(\mathrm{SiO}_{2}\right)_{n},(\mathrm{FeO})_{n},(\mathrm{MgS})_{n},(\mathrm{Si})_{n}$, and $(\mathrm{Fe})_{n}$ with $n=1-4$. We also include carbon chains up to $\mathrm{C}_{6}$ and benzene $\left(\mathrm{C}_{6} \mathrm{H}_{6}\right)$.

\section{Results and discussion}

Molecular abundances for the fully-mixed case are illustrated in Figure 1 for the PMSN surrogate. Species form at early times and in large amounts by non-steady state processes. The main formation and destruction chemical pathways are neutral-neutral reactions up to $\sim 600$ days whereas ion attack $\left(\mathrm{He}^{+}, \mathrm{O}^{+}\right)$and fast Compton electron destruction are effective at later times. Dominant molecules are $\mathrm{O}_{2}, \mathrm{CO}_{2}, \mathrm{SO}$, and $\mathrm{H}_{2}$ and $\mathrm{H}_{2} \mathrm{O}$ if hydrogen penetration from the progenitor envelope is allowed. The total molecular budget is $\sim 57 \mathrm{M}_{\odot}$ while it is $\sim 42 \mathrm{M}_{\odot}$ in the unmixed PMSN case (Cherchneff \& Lilly 2008). In any case, we find that 25 to $34 \%$ of the progenitor mass is ejected in molecular form in the local, pristine gas.

In CCSNe, molecular formation is also very efficient as illustrated in Figure 2. Again, main species include $\mathrm{O}_{2}, \mathrm{CO}_{2}, \mathrm{SO}, \mathrm{CO}$ and $\mathrm{NO}$, and $\mathrm{H}_{2}$ and $\mathrm{H}_{2} \mathrm{O}$ when $\mathrm{H}$-mixing is considered. In this specific case, $\sim 5 \%$ of hydrogen penetrate the helium core. $\mathrm{SiO}$ also forms in large amount but is quickly removed from the gas at $t \geqslant 550$ days due to its inclusion into dust precursors. The total molecular budget is $7.3 \mathrm{M}_{\odot}$, equivalent to $36.5 \%$ of the progenitor mass. As for the most abundant dust precursors., $\mathrm{AlO}$ and $\left(\mathrm{SiO}_{2}\right)_{2}$ start forming in large amounts at $t \geqslant 450$ days, a time in excellent agreement with the onset of dust formation in SN1987A (Lucy et al. 1989). This sequence indicates that corundum and quartz condense simultaneously at early times where the gas densities are high, leading to large dust amounts. Periclase, iron oxide and magnesium sulfite precursors form with large abundances but at much later times $(t \geqslant 750$ days $)$, therefore leading to small amounts of their parent condensates. This fully-mixed case thus fosters the formation of corundum and quartz, but hampers silicate formation like forsterite $\left(\mathrm{Mg}_{2} \mathrm{SiO}_{4}\right)$, for the abundance of periclase precursors is 4 orders of magnitude less than that of silica precursors at 650 days after explosion. These results disagree with condensation sequences 


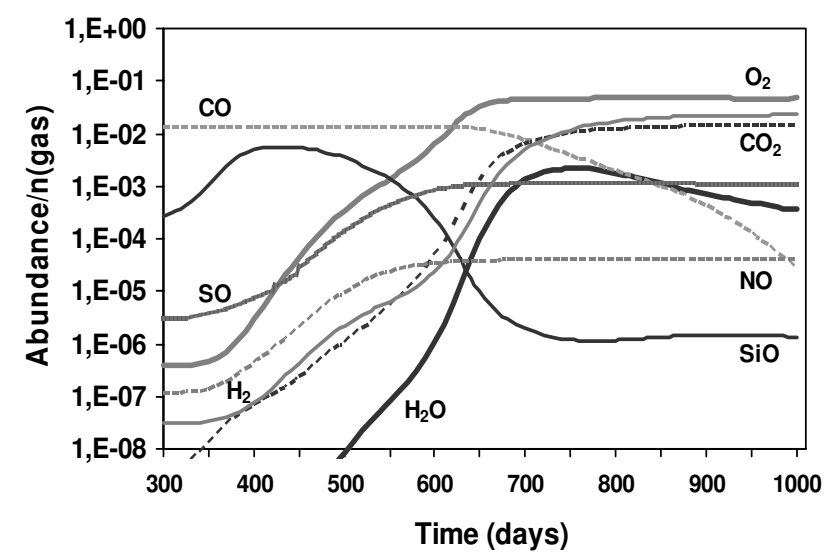

Figure 2. Molecular abundances versus time for the fully-mixed case of SN1987A.

derived by existing studies (Kozasa et al. 1989, 1991). These models predict the following condensate sequence for the fully-mixed case: corundum at 450 days, forsterite (or enstatite $\mathrm{MgSiO}_{3}$ ) at 550 days, and magnetite $\left(\mathrm{Fe}_{3} \mathrm{O}_{4}\right)$ at 630 days. This discrepancy points to the crucial role of chemical kinetics as the bottleneck to dust formation in circumstellar environments.

We conclude that SN ejecta are rich molecular environments. Their chemical composition depends on the degree of mixing of heavy elements in the helium core and of hydrogen from the progenitor envelope. Therefore molecules could be used as observational tracers of mixing in nearby SN ejecta. In the early universe, PMSN molecules may provide the cooling necessary to trigger Pop. II.5 star formation in the PMSN dense shell (Mackey et al. 2003, Salvaterra et al. 2004) if they survive the reverse shock some 10,000 years after the PMSN explosion.

\section{References}

Cherchneff, I. \& Lilly, S. 2008, ApJ (Letters), submitted

Clayton, D. D., Liu, W., \& Dalgarno, A. 1999, Science, 283, 1290

Donn, B. \& Nuth, J. A. 1985, ApJ, 288, 187

Douvion, T, Lagage, P. O., \& Pantin, E. 2001, AA, 369, 589

Kozasa, T., Hasegawa, H., \& Nomoto, K. 1989, ApJ, 344, 325

Kozasa, T., Hasegawa, H. \& Nomoto, K. 1991, AA, 249, 474

Lepp, S., Dalgarno, A., \& McCray, R. 1990, ApJ, 358, 262

Liu, W. \& Dalgarno, A. 1994, ApJ, 438, 789

Liu, W. \& Dalgarno, A. 1995, ApJ, 454, 472

Lucy, L. B., Danziger, I. J., Gouiffes, C., \& Bouchet, P. 1989, in: G. Tenorio-Tagle, M. Moles, \& J. Melnick (eds.), IAU Coll. 120, Structure and Dynamics of the Interstellar Medium (Berlin: Springer-Verlag), p. 164

Mackey, J., Bromm, V., \& Hernquist, L. 2003, ApJ, 586, 1

Nozawa, T, Kozasa, T., Umeda, H., Maeda, K., \& Nomoto, K. 2003, ApJ, 598, 785

Pinto, P. A. \& Woosley, S. E. 1988, Nature, 333, 534

Roche, P. F., Aitken, D. K., \& Smith, C. H. 1991, MNRAS, 252, 39

Salvaterra, R., Ferrara, A., \& Schneider, R. 2004, Nature, 10, 113

Schneider, R., Ferrara, A., Salvaterra, R. 2004, MNRAS, 351, 1379

Spyromilio, J., Meikle, W. P. S., Learner, R. C. M., \& Allen, D. A. 1988, Nature, 334, 327

Todini, P. \& Ferrara, A. 2001, MNRAS, 325, 726

Umeda, H. \& Nomoto, K. 2002, ApJ, 565, 385

Wooden, D. H., Rank, D. M, Bregman, J. D., Witteborn, F. C., Tielens, A. G. G. M., Cohen, M., Pinto, P. A., \& Axelrod, T. S. 1993, ApJS, 88, 477 


\section{Discussion}

UNKNOWN: I would think the isotropic signature maybe particularly telling about what is going on in the supernovae that you considered the CNO isotopes at all?

Cherchneff: Yes, I agree, but the chemistry so far does not include isotopes at all. I am just looking really at plain chemistry to try to identify which kind of molecules I can form.

UNKNOWN: Do these stars produce mostly 12-carbon and 16-oxygen?

CherchnefF: Yes, they do.

Mumma: I wonder if you consider the possibility of doing work on these molecules, water, $\mathrm{CO}$ and so forth in the infrared wavelengths before $\mathrm{mm}$ and submm facilities become available.

Cherchneff: In terms of observing molecules at the infrared wavelengths, this field was very active 20 years ago when SN1987A exploded. If you look at the conditions in the ejecta of supernovae where dust forms, they are not so different from the conditions you find in AGB stars very close to the photosphere. We know that in AGB winds, dust forms along with large amounts of molecules. It is very much the same in supernovae. The problem with the infrared is that you really need to observe 'warm' molecules right after the explosion. On the other hand, if you look at objects that exploded two or three years ago at submm wavelengths, you should see molecules in the ejecta.

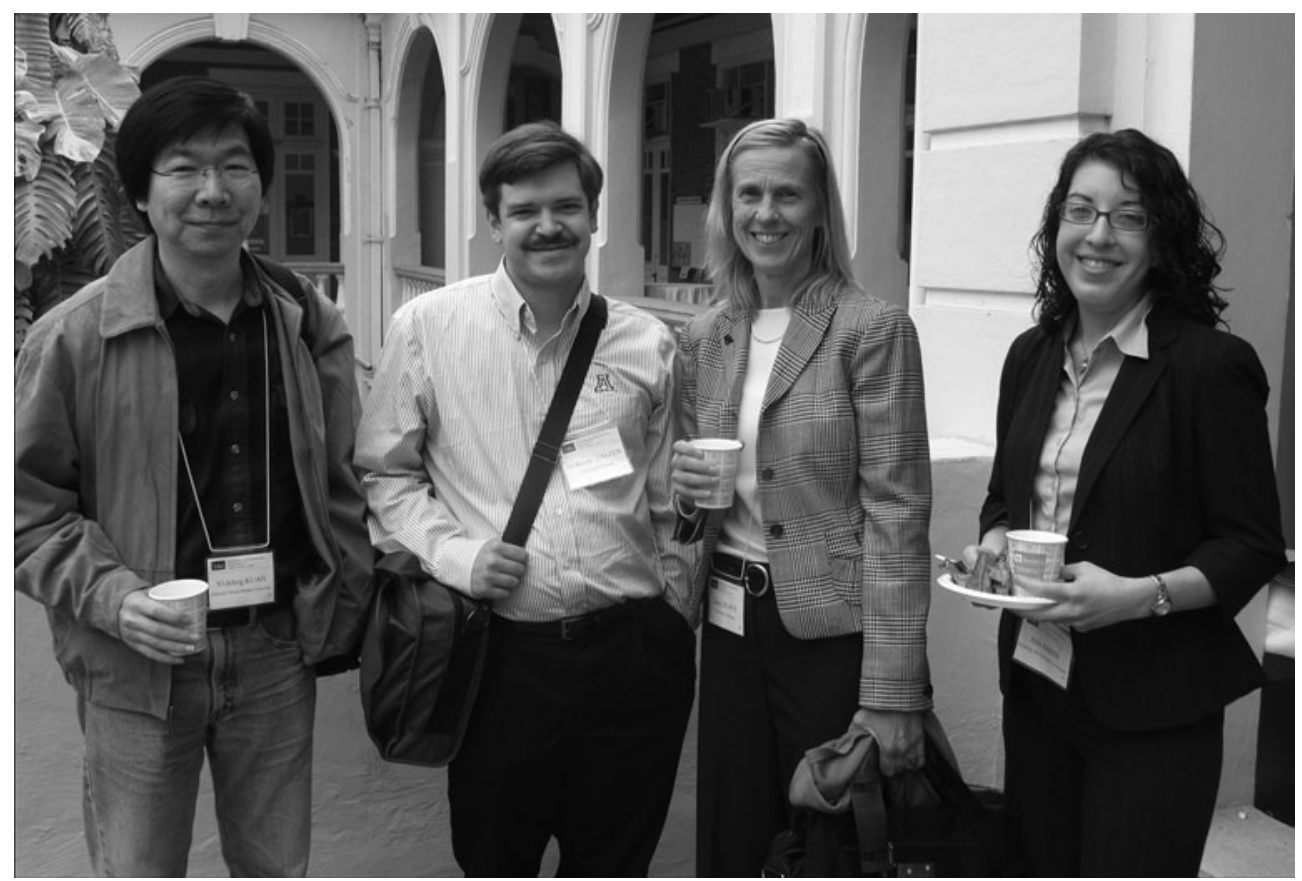

From let to right: Yi-Jehng Kuan, DeWayne Halfen, Lucy Ziurys, Erin Smith (photo by Dale Cruikshank). 


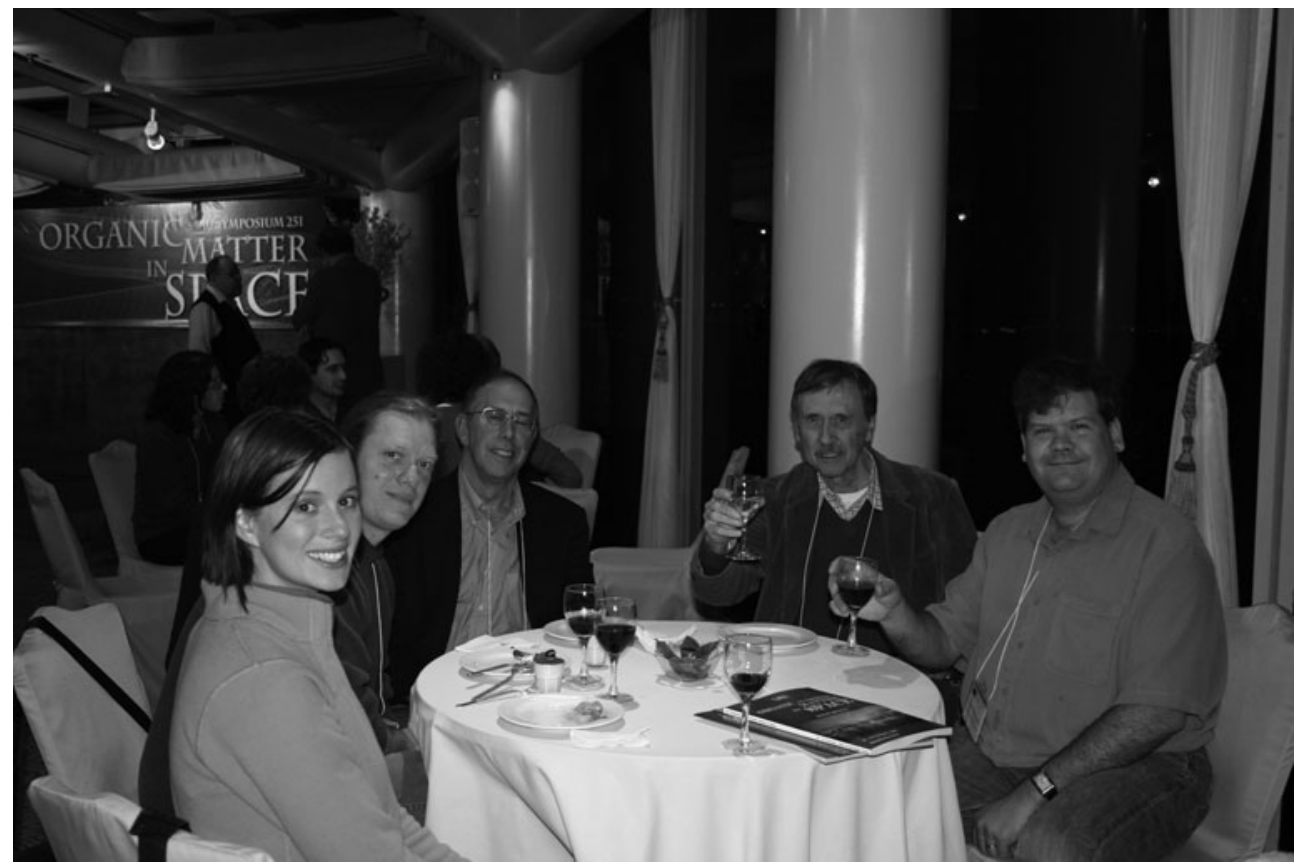

Welcome reception. From left to right: Kasandra O'Malia, Oscar Martinez, Ted Snow, Ernst Zinner, Larry Nittler. 